\title{
Analysis on the Quality Factors of Improving Highway Survey and Design
}

\author{
Miao Chengtao \\ Dezhou Road Survey and Design Institute, Shandong Province, Shandong Dezhou, 253077
}

Keywords: highway; design quality; quality factor

\begin{abstract}
This paper mainly from the perspective of the actual project analysis, taking the author's previous participation in an engineering project as an example, describes the highway survey and design steps and discusses them from the three directions of optimization design, project action plan formulation and site verification. At last, it describes the quality factors of highway survey and design, and analyzes them in detail from different angles, so as to provide reference for improving the quality analysis and exploration of highway survey and design.
\end{abstract}

\section{Introduction}

With the rapid development of social economy of our country, the scale of various infrastructures continues to expand, especially as the construction of expressways continues to prolong. At the same time, with the needs of social development, the exploration design and quality requirements have received extensive attention when implementing highway construction. Therefore, we must attach great importance to the issue of survey and design quality, and based on the quality factors, accurately control the basic elements of management, science and technology, and talents, and effectively innovate and integrate them, so as to provide effective guarantees for improving the quality of highway survey design.

\section{Analysis of Project Profile}

Wucheng County Road Network Renovation and Improvement Project - X025 Yuan Road (Jinguang Avenue to Xingkou Bridge) are located in the northwest of Wuzhou County in Dezhou City. The starting point of this design section is at the intersection of X025 Zhenghao Road and Jinguang Avenue, and goes straight along the ancient road to the west. After passing through the Luteng community, it passed through the German-Shangshan highway and continued to the west through Nanzhencunbei. It finally reached the south bridgehead of the Xingkou Bridge and took the Zhengkou Bridge. The total length of the route is $7.19 \mathrm{~km}$. The old roads of $\mathrm{K} 0+00-\mathrm{K} 0+510$ and $\mathrm{K} 1+457.9-\mathrm{K} 2+438$ of this section are cement pavements, and the shoulder width of the soil is $0.5 \mathrm{~m}-2.5 \mathrm{~m}$; the old roads of $\mathrm{KO} 0+510-\mathrm{K} 1+457.9$ and $\mathrm{K} 2+438-\mathrm{K} 7+190.31$ are asphalt roads. The width of the road is $6 \mathrm{~m}$, and the shoulder width of the dirt road is $0.5 \mathrm{~m}-2.5 \mathrm{~m}$. Plane linear indicators basically meet the three-way standard, but there are more small detours. Crossing villages and towns along multiple sections of the road will cause some disturbance to the traffic. The roadbed is stable and basically free from disease. Due to the low standard of initial construction, especially the rapid growth of traffic in recent years, there are many overloaded vehicles, and the existing roads have serious road surface diseases, such as cracks, pits, and other diseases. The vehicles are crowded, the traffic capacity is deficient, and the overall service level is relatively low, which cannot meet the needs of local economic development. The transformation of this project can improve Zheng Hao Road's capacity and service level, improve driving conditions, ease traffic pressures, optimize the road network layout in the region, and further strengthen the links between villages along the route. Besides, it is of great significance to promote the economic and social development along the line. 


\section{Analysis of Highway Survey and Design Procedures}

\subsection{Optimization Design of Construction Drawings}

In the process of designing construction drawings, the pre-engineering design plan is the benchmark. In the earliest design stage, the basis for different approval should be effectively compared, and the project construction funds, construction scale, and actual conditions of the construction project should be taken into consideration, so as to effectively adjust and optimize the construction plan and line plan. However, because of the relatively high difficulty of the overall construction of elevated roads, and its own particularities. Therefore, when conducting surveys on the construction site, it is necessary to carry out in-depth analysis and optimization of the design plan, and to focus on the actual application of indicators and route plans to compare special lines, then focus on Analyze and study, and combine the design requirements of the typical project and local route plan Alibaba based on optimization, thus guaranteeing its rationality and scientific design.

\subsection{Formulation of Project Action Plan}

According to the actual situation and characteristics of the project, it is necessary to analyze and contrast the existing data information and approval opinions, especially the difficulties and priorities of the project. Through the survey design, the whole highway design can be guided and optimized. Therefore, definite plan compares the overall organizational form, technical design book, and the work program to conduct thein-depth analysis. Especially in terms of schedule formulation, work stage division, and manual division of labor design, we must attach great importance to the issue of design quality and conduct strict comparison and review of relevant data, so as to enhance the control of relevant data and further guarantee application efficiency. For mechanical design books, it is necessary to clarify the current status of the utilization and collection of relevant utilization data, and conduct a comprehensive review of it in accordance with the actual auditing standards so as to ensure that it meets the survey design standards.

\subsection{Survey Design Site Verification Analyses}

In the process of the construction of highway construction, due to the differences in geological environment in different regions, when implementing survey and design, it is necessary to attach great importance to the design of links and further implement the survey and design work according to the specific design standards already implemented. First, it is necessary to design and survey the route areas planned in the early stage, and to adjust the exploration area in light of the complex local geological conditions, and to assure the effectiveness of the survey design through the expansion method.Secondly, when carrying out the geological conditions exploration design and analysis, the quantitative analysis of the implementation of a large amount of work, and the geology of the complex areas to be followed to ensure the degree of control, thereby enhancing the quality of the survey design, to provide protection for the implementation of the entire project sequence.

\section{Analysis on Quality Factor of Highway Survey Design}

\subsection{Analysis of Management Factors in High-level Highway Survey and Design Quality}

Management factors play animportant role in the implementation of highway survey and design. First of all, in the process of strengthening the design quality management, we must avoid the problems of exploration and design in terms of technology, systems, etc. In this regard, we must adjust the various aspects of engineering design, from the management to the entire exploration and design process, and promote different links. Improved connectivity. Secondly, it should be carried out in compliance with standardized management system standards. Through management representatives, different functional departments and management departments should be assigned division of labor to clear their own responsibilities. At the same time, the impact of management effectiveness may be analyzed, and analysis of control criteria and statistics literal form controls the 
survey design process in an all-round way so as to improve the quality of the entire survey design. Finally, in the process of carrying out survey and design, it is necessary to accurately control the design status of the survey according to the management system, and to analyze and provide no application elements according to the overall survey and design standards, so as to ensure that the entire design mechanism is under the condition of accurate control.

\subsection{Analysis of Technological Factors in the Exploration and Design Quality of Highway.}

In the implementation of highway survey and design, technology play an important role and significance in improving its integral quality. In the process of controlling highway quality, it is necessary to prepare the corresponding software platform and hardware equipment in advance. First, in the initial design period, various scientific and technological factors must be accurately assessed, and the degree of emphasis on scientific and technological factors should be increased. Some key technical equipment should be introduced according to the need of the period. Secondly, based on the new network technology, the controlled software platform must be based on the latest network technology to improve the efficiency of data information transmission and ensure the validity of data information evaluation and assessment including GIS, GPS and RS. In the actual exploration process, In accordance with high-density electrical methods, reflections, seismic refraction and other related technical indicators to review the indicators, especially the large-scale field survey work, and more emphasis on the error of the segment coordinates, so the specific vegetation in the highway construction area must be specific Analysis, and in accordance with the characteristics of the specific implementation point and line, to adjust the late printing changes, and thus promote the improvement of the entire measurement accuracy, improve the quality and level of the entire highway construction design.

\subsection{Analysis of Talents in the Quality of Highway Exploration and Design}

In the implementation of the highway survey and design process, it is necessary in order to include different levels and different professional personnel, so as to provide effective protection for the entire exploration and design quality. As a survey and design personnel, when participating in the survey and design work, it is necessary to analyze the design form in depth and control the optimal design points. Therefore, relevant practitioners must have abundant theoretical knowledge and professional skills. When implementing the inclusive design stage, they must compare the management plan and economic forms, so as to highlight the entire survey design points. Since the survey and design work is always intuitive for the quality of the entire highway, it is necessary to attach great importance to the application of related talents, so that personnel can have a full understanding of the entire survey and design process, and have the flexibility to grasp and know the details of the various exploration links and surveys, and thus the entire high speed. Highway survey and design quality provide effective protection.

\section{Conclusion}

In summary, during the implementation of expressway survey and design, due to its own particularities, in the actual implementation process, it is necessary to ensure that it can meet the design standards in accordance with the geological conditions of the entire project construction area and the later changes. At the same time, due to the construction of expressway construction projects and the relatively large amount of works, they must be strictly implemented in accordance with design standards to prevent them from being affected by bad external factors, in accordance with the actual design conditions, to plan the entire survey and design standards, so as to effectively improve the highway survey and design quality guarantees the quality of the entire project and its service life.

\section{References}

[1] Li Dinggang. On the overall design of the highway and its management approach [J]. Urban 
Construction Theory: Electronic Edition, 2015(23) :7037-7037

[2] Meng Fanlong, Huang Yujie, Xie Shibiao et al. Investigation and analysis of the quality of subgrade work under construction in Heilongjiang Province[J].Heilongjiang Communications Science and Technology,2011(9) :111-111

[3] Song Yu. Application Research of ISO9001 Quality Management System in Highway Engineering Survey [J]. China Real Estate, 2015(15):154-154

[4] Zhang Jian. Characteristics of investigation and design of key sections of the Weiyang Plain section of the Chang-Chang Expressway and measures for key technical problems [J]. Research on Theory of Urban Construction: Electronic Edition, 2012(17):12-13. 